\title{
Sarcoidosis Resulting in Exsanguinating Esophageal Variceal Hemorrhage
}

Adam Blumenberg, MD, MA; Sophia Sharifali, MD; Richard Sinert, DO

\section{A 47-year-old woman with a history of pulmonary and renal sarcoidosis presented for evaluation of hematemesis and melena.}

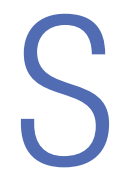

arcoidosis is a systemic disorder of unknown etiology and is characterized by the formation of granulomas throughout various organs in the body. The most common form is pulmonary sarcoidosis, which affects $90 \%$ of patients; the second most common form is oculocutaneous sarcoidosis; ${ }^{1}$ and the third most common form is hepatic sarcoidosis, which affects $63 \%$ to $90 \%$ of patients. ${ }^{2}$ Although the liver is frequently involved in all forms of sarcoidosis, only a fraction of patients present with clinically evident liver disease. ${ }^{1}$ Approximately $20 \%$ to $30 \%$ of patients have abnormalities on liver function tests, whereas only about $1 \%$ of patients show evidence of portal hypertension and cirrhosis. ${ }^{3}$ In fact, in the English literature, there were 35 reported cases of portal hypertension due to sarcoidosis between 1949 to 2001, of which 16 of the patients had no evidence of cirrhosis. ${ }^{4}$

The diagnosis of sarcoidosis is usually made by a compilation of clinical signs and symptoms, imaging studies, and biopsies demonstrating noncaseating granulomas. This case report describes a patient who presented with portal hypertension and esophageal variceal bleeding secondary to sarcoidosis of the liver without cirrhotic changes.

\section{Case}

A 47-year-old woman presented to the ED via emergency medical services with a 1-hour history of hematemesis and melena. The patient stated that she felt fatigued, nauseated, and light-headed, but had no pain or focal weakness. Her medical history was significant for pulmonary and renal sarcoidosis. She underwent a liver biopsy 1 week prior to presentation, with a 6-day hospitalization period, due to new ascites found on examination.

The patient's vital signs at presentation were: blood pressure (BP), 72/56 mm Hg;

\footnotetext{
Dr Blumenberg is a resident physician, department of emergency medicine, Kings County Hospital Center, Brooklyn, New York; and department of emergency medicine, Downstate Medical Center, Brooklyn, New York. Dr Sharifali is a resident physician, department of emergency medicine, Kings County Hospital Center, Brooklyn, New York; and department of emergency medicine, Downstate Medical Center, Brooklyn, New York. Dr Sinert is an attending physician, department of emergency medicine, Kings County Hospital Center, Brooklyn, New York; and department of emergency medicine, Downstate Medical Center, Brooklyn, New York.
}

Authors' Disclosure Statement: The authors report no actual or potential conflict of interest in relation to this article. 
Table. Patient's Laboratory Values

\begin{tabular}{|c|c|c|c|}
\hline Value & 1 Week Prior to Presentation & At Presentation & Reference Range \\
\hline Sodium & 139 & 131 & $135-146 \mathrm{mmol} / \mathrm{L}$ \\
\hline Potassium & 4.6 & 6.9 & $3.5-5.0 \mathrm{mmol} / \mathrm{L}$ \\
\hline Chloride & 106 & 99 & 98-106 mmol/L \\
\hline Carbon dioxide & 13 & 7 & $24-31 \mathrm{mmol} / \mathrm{L}$ \\
\hline Blood urea nitrogen & 74 & 138 & $6-20 \mathrm{mg} / \mathrm{dL}$ \\
\hline Creatinine & 2.9 & 5.4 & $0.5-0.9 \mathrm{mg} / \mathrm{dL}$ \\
\hline Glucose & 96 & 160 & $70-99$ mg/dL \\
\hline Calcium & 8.2 & 7.7 & $8.6-10.0 \mathrm{mg} / \mathrm{dL}$ \\
\hline Protein & 5.1 & 5.1 & $6.0-8.5 \mathrm{~g} / \mathrm{dL}$ \\
\hline Albumin & 2.3 & 2.2 & $2.8-5.7 \mathrm{~g} / \mathrm{dL}$ \\
\hline Aspartate aminotransferase & 36 & 25 & $10-35 \mathrm{U} / \mathrm{L}$ \\
\hline Alanine transaminase & 18 & 14 & 0-31 U/L \\
\hline Alkaline phosphatase & 390 & 497 & $25-125 \mathrm{U} / \mathrm{L}$ \\
\hline T bilirubin & 0.45 & 0.45 & $0.0-1.2 \mathrm{mg} / \mathrm{dL}$ \\
\hline Platelets & 256 & 400 & $130-400 / n L$ \\
\hline Hemoglobin & 7.7 & 4.7 & $12.0-16.0 \mathrm{~g} / \mathrm{dL}$ \\
\hline Hematocrit & 26.3 & 17.1 & $37.0-47.0 \%$ \\
\hline White blood cells & 9.3 & 18.4 & 4.5-10.9/nL \\
\hline International normalized ratio & 1.1 & 1.1 & $0.8-1.2$ \\
\hline Activated partial thromboplastin time & 27.0 & 27.3 & $22-29 \mathrm{sec}$ \\
\hline
\end{tabular}

heart rate (HR), 133 beats/min, respiratory rate, 24 breaths/min; and temperature, $97.0^{\circ} \mathrm{F}$. Oxygen saturation was $99 \%$ on room air. Physical examination revealed an alert and oriented middle-aged woman in extremis who was vomiting dark-colored blood. The cardiac and pulmonary examination revealed no extraneous sounds; the abdominal examination showed ascites with a liver edge palpable $4 \mathrm{~cm}$ beneath the right costal margin. The patient had no scleral icterus, palmar erythema, spider angiomata, fetor hepaticus, caput medusa, cutaneous ecchymoses, or any other stigmata of cirrhosis.

Two large-bore peripheral intravenous (IV) catheters were placed and a massive blood transfusion protocol was initiated. Packed red blood cells (PRBCs) from the resuscitation-area refrigerator were infused immediately via a pressurized fluid warmer.

After consultation with gastroenterology and general surgery services, the patient 


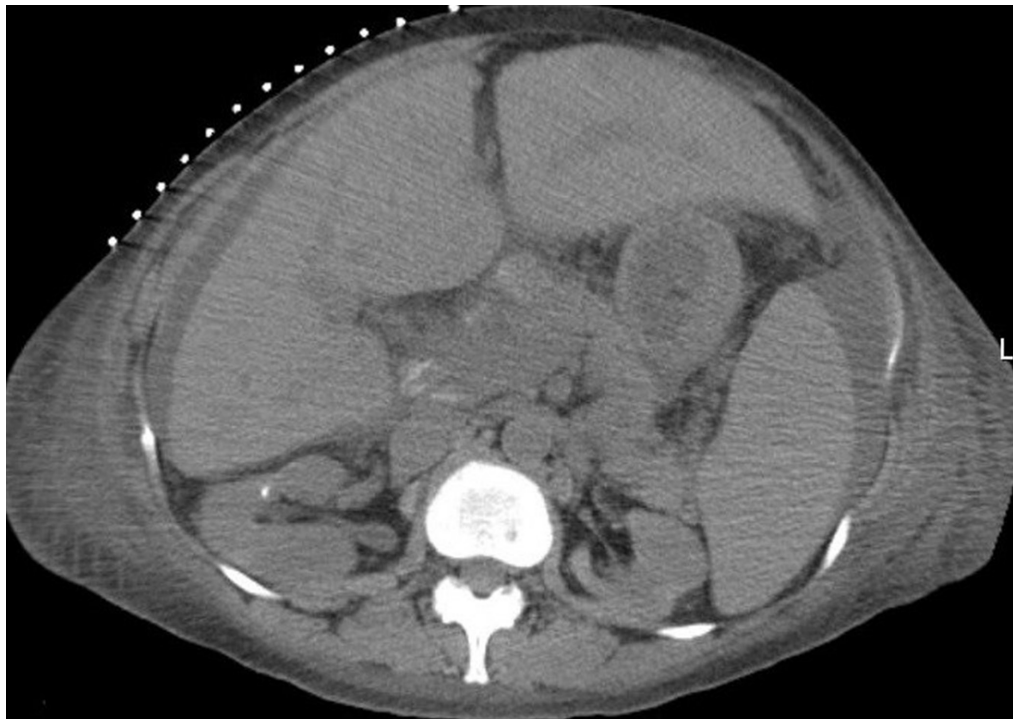

Figure 1. Computed tomography scan of the patient's abdomen and pelvis demonstrating ascites and hepatomegaly with scattered hypoattenuating lesions and periportal lymphadenopathy. plasma, and platelets over a 3-hour period. During transfusion, the patient's vital signs improved to a systolic BP ranging between 110 to $120 \mathrm{~mm} \mathrm{Hg}$ and an HR ranging between 90 to 110 beats/min; she did not experience any further hypotensive episodes throughout her stay in the ED.

Laboratory studies were significant for metabolic acidosis, hyperkalemia, acute on chronic anemia, leukocytosis, and acute on chronic renal failure. Synthetic function of the liver and transaminases appeared normal (Table).

The patient's hyperkalemia was treated with $1 \mathrm{~g}$ calcium chloride IV, $50 \mathrm{~g}$ dextrose IV, and $10 \mathrm{U}$ regular insulin IV. A portable chest radiograph showed an appropriately positioned endotracheal tube, and an electrocardiogram revealed sinus tachycardia without signs of hyperkalemia. A computed tomography (CT) scan of the abdomen and pelvis from the patient's recent hospitalization, 1 week prior to presentation, showed hepatomegaly, liver granulomas, ascites, and periportal lymphadenopathy (Figure 1).

A review of the patient's recent liver biopsy and ascitic fluid analysis revealed noncaseating granulomas compressing the hepatic sinusoids, and a serum ascites albumin gradient greater than $1.1 \mathrm{~g} / \mathrm{dL}$, implying portal hypertension without cirrhosis. The surgical team attempted to place a Sengstaken-Blakemore tube, but the device could not be positioned properly due to the patient's narrowed esophagus.

The ED nurses cleaned the patient, preserving her dignity; thereafter the patient's adult children visited with her briefly before she was taken for an upper endoscopy, which was performed in the ED. The endoscopy revealed actively hemorrhaging esophageal varices at the gastroesophageal junction (Figure 2). The varices were treated with endoscopic ligation; the gastroenterologist placed a total of 11 bands, resulting in cessation of bleeding.

After the endoscopy, the patient was admitted to the medical intensive care unit 
(ICU). Approximately 1.5 hours after arriving at the ICU, she developed renewed hematemesis. Despite efforts to control bleeding and provide hemodynamic support, the patient died 1 hour later.

\section{Discussion}

\section{Etiology}

Esophageal variceal hemorrhage is caused by pressure elevation in the portal venous system, leading to engorged esophageal veins that can bleed spontaneously. Approximately $90 \%$ of portal hypertension is due to liver cirrhosis. ${ }^{5}$ The remaining $10 \%$ of cases are primarily vascular in etiology, with endothelial dysfunction and thrombosis leading to increased portal resistance. Noncirrhotic causes of portal hypertension include malignancy, congenital diseases, viral hepatitides, vascular thromboses or fistulae, constrictive pericarditis, fatty liver of pregnancy, drugs, radiation injury, and infiltrative diseases. ${ }^{5}$

Sarcoidosis may cause noncaseating granulomas to form in the liver, leading to portal hypertension and fatal exsanguination from esophageal variceal hemorrhage. Although the lesions of sarcoidosis classically form in the lungs, any organ system may be affected..$^{6,7}$ Frank cirrhosis of the liver occurs in only $1 \%$ of sarcoidosis patients; however, radiographic involvement of the liver is seen in $5 \%$ to $15 \%$ of patients. ${ }^{8}$

There are several mechanisms which may be responsible for portal hypertension in patients with sarcoidosis, including granulomas causing mass effect on the hepatic sinusoids; arteriovenous shunts within the granuloma; granulomatous phlebitis within the sinusoids; or compressive periportal lymphadenopathy. ${ }^{9}$ Regardless of the mechanism, a review of the literature demonstrates an association between sarcoidosis and symptomatic portal hypertension., ${ }^{2,4,10,11}$

Although our patient ultimately died, early initiation of massive blood transfusion protocol, airway protection, attention to electrolytes, and endoscopic control of the hemorrhage source provided the best

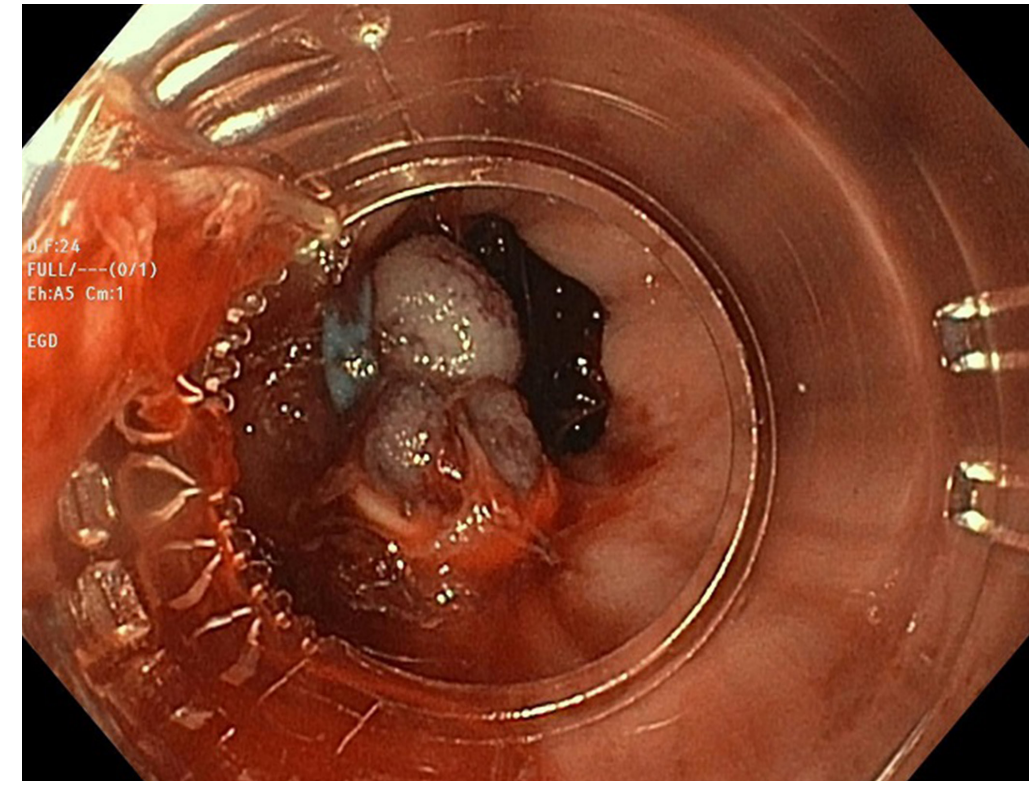

Figure 2. Endoscopy demonstrating ligation of the patient's esophageal varices.

chance for survival.

\section{Medical Therapy}

The first priority in managing and treating esophageal varices is to secure the patient's airways to prevent aspiration. Two large bore IV lines should be placed to permit rapid infusion of crystalloid fluids or blood products. Initiating antibiotics, specifically IV ceftriaxone, to patients with variceal bleeding is a class I recommendation, as this is the only intervention shown to increase patient survival. ${ }^{12}$ Although proton pump inhibitors (PPI) and somatostatin analogues (typically octreotide) are frequently given, they are both class II recommendations because there is limited evidence supporting the benefit of their use. ${ }^{12}$ However, current guidelines recommend treating patients for variceal bleeding with an initial bolus of a PPI, followed by a continuous infusion of PPI for 72 hours. As previously noted, multiple studies, have failed to show any decrease in mortality associated with this treatment. ${ }^{12}$

Other agents that are used to treat variceal bleeding include octreotide and vasopressin. Octreotide, a somatostatin analog, 
is generally given as an initial IV bolus followed by continuous infusion, and has been shown to decrease transfusion requirements without mortality benefit. ${ }^{12}$ Vasopressin is generally given to critically ill patients, and is considered a third-line treatment for variceal bleeding.

Since our patient had a history of chronic kidney disease, desmopressin was empirically administered in the event platelet dysfunction was a contributing factor to bleeding. ${ }^{13}$ The absence of cirrhosis was significant because our patient was unlikely to have a bleeding diathesis caused by coagulation factor deficiency. Therefore, the goal transfusion ratio of blood products should be balanced, similar to that in traumatic exsanguination, rather than favoring an increased ratio of plasma to other blood products. Similarly, tranexamic acid was administered because insufficient tamponade rather than coagulopathy was the presumed cause of sustained hemorrhage.

An additional complicating factor in our patient's care was the potential effect of the massive transfusion on electrolytes. Packed RBCs have a pH of approximately 6.8 and may carry up to $25 \mathrm{mmol} / \mathrm{L}$ of potassium, which may have exacerbated our patient's underlying hyperkalemia. ${ }^{14}$ Rapid blood transfusion also places patients at risk for acute hypocalcemia secondary to citrate toxicity; this did not occur in our patient in part because the metabolic function of her liver was preserved and citrate could be broken down in the hepatocyte Krebs cycle..$^{15}$ Calcium therapy doubled as treatment for the hyperkalemia and as prophylaxis against further hypocalcemia. No dysrhythmias were observed.

\section{Surgical Intervention}

Emergency physicians should consult with gastroenterology services so that an endoscopy can be performed as soon as possible to evaluate for and control bleeding. When an endoscopy cannot be performed rapidly, there are multiple balloon tamponade devices available that can be used to temporize the bleeding, such as the Sengstaken-Blakemore tube. ${ }^{12}$

Although balloon tamponade devices are typically reserved for the last line of therapy, endoscopy rather than transjugular intrahepatic portosystemic shunt (TIPS) was the preferred method of hemorrhage source control in our patient for several reasons. First, although the working diagnosis of varices was based on the patient's history, we wanted to evaluate for other causes of upper gastrointestinal bleeding since our patient had no history of endoscopy. Therefore, endoscopy had both a therapeutic and diagnostic value. Secondly, though TIPS may decrease pressure within the bleeding varix, only endoscopy permits direct hemostasis. Also, endoscopy also was preferred over TIPS because our patient was too unstable to move to the interventional radiology suite. ${ }^{16}$

\section{Conclusion}

Although life-threatening esophageal variceal hemorrhage is a rare manifestation of an uncommon disease, it should be considered in the differential diagnosis of a patient who has sarcoidosis and presents with gastrointestinal bleeding. Additionally, when caring for a patient with massive hematemesis without evidence of liver cirrhosis, other etiologies of portal hypertension and esophageal varices, such as sarcoidosis, should be considered.

\section{References}

1. Rao DA, Dellaripa PF. Extrapulmonary manifestations of sarcoidosis. Rheum Dis Clin North Am. 2013;39(2):277-297. doi:10.1016/j.rdc.2013.02.007.

2. Mistilis SP, Green JR, Schiff L. Hepatic sarcoidosis with portal hypertension. Am J Med. 1964;36(3):470475. doi:10.1016/0002-9343(64)90175-5.

3. Tekeste H, Latour F, Levitt RE. Portal hypertension complicating sarcoid liver disease: case report and review of the literature. Am J Gastroenterol. 1984;79(5):389-396.

4. Ivonye C, Elhammali B, Henriques-Forsythe M, Bennett-Gittens R, Oderinde A. Disseminated sarcoidosis resulting in portal hypertension and gastrointestinal bleeding: a rare presentation. Can J Gastroenterol. 2012;26(8):508-509. http://www.ncbi.nlm.nih.gov/ pubmed/22891173. Accessed May 16, 2018.

5. Tetangco EP, Silva RG, Lerma EV. Portal hypertension: etiology, evaluation, and management. Dis Mon. 2016;62(12):411-426. doi:10.1016/ 
j.disamonth.2016.08.001

6. Valeyre D, Prasse A, Nunes H, Uzunhan Y, Brillet PY, Müller-Quernheim J. Sarcoidosis. Lancet. 2014;383(9923):1155-1167. doi:10.1016/S01406736(13)60680-7.

7. Al-Kofahi K, Korsten P, Ascoli C, et al. Management of extrapulmonary sarcoidosis: challenges and solutions. Ther Clin Risk Manag. 2016;12:1623-1634. doi:10.2147/TCRM.S74476.

8. Iannuzzi MC, Rybicki BA, Teirstein AS. Sarcoidosis. N Engl J Med. 2007;357(21):2153-2165. doi:10.1056/ NEJMra071714.

9. Ebert EC, Kierson M, Hagspiel KD. Gastrointestinal and hepatic manifestations of sarcoidosis. Am J Gastroenterol. 2008;103(12):3184-3192. doi:10.1111/ j.1572-0241.2008.02202.x

10. Fraimow W, Myerson RM. Portal hypertension and bleeding esophageal varices secondary to sarcoidosis of the liver. Am J Med. 1957;23(6):995-998.

11. Saito H, Ohmori M, Iwamuro M, et al. Hepatic and gastric involvement in a case of systemic sarcoidosis presenting with rupture of esophageal varices.
Intern Med. 2018;56(19):2583-2588. doi:10.2169/ internalmedicine.8768-16.

12. DeLaney M, Greene CJ. Emergency Department evaluation and management of patients with upper gastrointestinal bleeding. Emerg Med Pract. 2015;17(4):1-18; quiz 19.

13. Ozgönenel B, Rajpurkar M, Lusher JM. How do you treat bleeding disorders with desmopressin? Postgrad Med J. 2007;83(977):159-163. doi:10.1136/ pgmj.2006.052118.

14. Sümpelmann R, Schürholz T, Thorns E, Hausdörfer J. Acid-base, electrolyte and metabolite concentrations in packed red blood cells for major transfusion in infants. Paediatr Anaesth. 2001;11(2):169173. doi:10.1046/j.1460-9592.2001.00637.x.

15. Monchi M. Citrate pathophysiology and metabolism. Transfus Apher Sci. 2018;56(1):28-30. doi:10.1016/j. transci.2016.12.013.

16. Shah RP, Sze DY. Complications during transjugular intrahepatic portosystemic shunt creation. Tech Vasc Interv Radiol. 2016;19(1):61-73. doi:10.1053/j. tvir.2016.01.007. 